\title{
ALTERAÇÕES ELETRENCEFALOGRÁFICAS EM PACIENTES COM HIPERPLASIA SUPRA-RENAL CONGÊNITA SECUNDÁRIA À DEFICIÊNCIA DA 21- HIDROXILASE
}

\author{
MIRIAM DA COSTA OLIVEIRA*, PAULO HENRIQUE GONZÁLES* * \\ MARIA HELENA FERNANDES ${ }^{* * *}$, HENRY WOLFF****
}

RESUMO - Alteraçðes eletrencefalográficas têm sido detectadas em crianças com pubarca precoce. Esse achado poderia ser o reflexo da presença atual ou passada de patologia do sistema nervoso central ou consequencia do nivel aumentado de esteróides sexuais. Com o objetivo de testar essa última hipótese, submetemos à avaliaçăo eletrencefalográfica 10 pacientes $(7 \mathrm{~F}, 3 \mathrm{M})$ com hiperplasia supra-renal congênita (HSRC) por deficiência da 21-hidroxilase, 4 deles com a forma clássica e $6 \mathrm{com}$ a forma não-clássica da doença. $O$ exame neurológico tradicional foi realizado em 7 pacientes, sendo normal em todos, enquanto o exame neurológico evolutivo diagnosticou distúrbio de hiperatividade e déficit de atenção $\mathrm{em}$ dois. Em outro pacicnte, dados de história foram compativeis com retardo do desenvolvimento neuropsicomotor. A avaliaçđo cletrencefalográfica, quantitativa com mapeamento cerebral nos pacientes com idade superior a 3 anos $(n=9)$, e tradicional no restante, foi considerada anormal para a idade em 8 pacientes $(80 \%)$, sendo o traçado $\mathrm{cm} 7$ casos caracterizado cono lento. A alta frequência de alteraçסes eletrencefalográficas nessa amostra, bem como na pubarca precoce, sugere que esses achados sejam secundários à presença de niveis androgênicos elevados em faixa etária inapropriada e alerta para a importância da avaliaçð̆o $c$ acompanhamento neurológico de crianças portadoras de HSRC.

PALAVRAS-CHAVE: eletrencefalograma, csteróides sexuais, hiperplasia supra-renal congênita, deficiência de 21 -hidroxilase.

Electroencephalographic changes in patients with congetinal adrenal hyperplasia due to 21-hydroxylase deficiency

ABSTRACT - Electroencephalographic changes have been detected in children with premature pubarche. These findings might be the result from past or current central nervous system dysfunction, or from increased sex steroids. In order to test the last assertion we have performed electroencephalographic studies in 10 patients ( $7 F$, $3 \mathrm{M}$ ) with congenital adrenal hyperplasia (CAI) due to 21-hydroxylase deficiency, of whom 4 classic and 6 nonclassic forms of the syndrome. Traditional neurologic cxamination was performed in 7 patients, the result being considered normal in everyone, while evolutional neurologic cxamination detected hyperactive disturbance and attention deficit in two patients. In another patient, the clinical findings were compatiblc with delay of neuropsychomotor development. Quantitative electroencephalography with brain mapping in paticnts over three years-old $(n=9)$, and conventional EEG in the rcmaining patient, were considered abnormal for age in 8 patients $(80 \%)$, the record being characterized as slow in 7 patients. The high rate of electroencephalographic changes in this sample, as well as in premature pubarche, suggests that these findings might result from high inappropriate for age androgenic levels, and warns about the importance of neurologic examination and follow-up in children with CAIl.

KEY WORDS: clectroencephalogram, scxual stcroids, congcnital adrenal hyperplasia, 21 -hydroxylase deficiency.

Estudo desenvolvido nas Disciplinas de Endocrinologia c de Neurologia da Fundaçðo Faculdade Federal de Ciências Médicas de Porto Alegre (FFFCMPA): *Professora Adjunta Doutora da Disciplina de Endocrinologia; **Médico Residente da Disciplina de Endocrinologia; ***Professora Auxiliar da Disciplina de Neurologia; ****Professor Titular da Disciplina de Endocrinologia. Aceite: 15-dezembro-1995. 
Há 30 anos, Liu e col. relataram a presença de alterações eletrencefalográficas de padrão inespecífico em $80 \%$ de uma série de 42 crianças com puberdade verdadeira idiopática ${ }^{8}$. Recentemente, um novo estudo avaliando os achados eletrencefalográficos em uma série de 15 crianças com várias apresentações de precocidade sexual detectou alterações em $66 \%$ da amostra, incluindo os 5 casos de pubarca precoce ${ }^{13}$. Esses achados poderiam estar relacionados a lesð̃es neurológicas não diagnosticadas pelos métodos convencionais, ou serem consequentes ao hiperandrogenismo em faixa etária precoce. Para testar a última hipótese foi realizado o presente estudo, que objetiva a investigação de alterações eletrencefalográficas em crianças com hiperplasia supra renal congênita (HSRC) por deficiência da 21- hidroxilase.

\section{PACIENTES E MÉTODOS}

Foram avaliadas prospectivamente 10 crianças com HSRC, 7 do sexo feminino e 3 do masculino, que consultaram no Serviço de Endocrinologia da FFFCMPA/ISCMPA. Em 6 pacientes foi diagnosticada a forma não-clássica da HSRC por deficiência da 21- hidroxilase e, em 4, a forma clássica, sendo em 2 casos perdedora de sal.

$\mathrm{Na}$ avaliação do crescimento e estadiamento puberal foram utilizadas as curvas de Tanner $^{16}$. O exame neurológico tradicional e o evolutivo foram efetuados pelo mesmo neuropediatra.

A radiografia de mãos e punhos para avaliação da idade óssea foi interpretada conforme Greulich e Pyle $^{4}$, sendo considerado como avanço na idade óssea um valor superior a 2 desvios-padrăo da média.

Das dosagens hormonais constaram a 17-hidroxiprogesterona (17OHP) em nivel basal e aos 30 e 60 minutos após a administração intravenosa de $250 \mathrm{mcg}$ de 1-24 ACTH (Cortrosina), o sulfato de deidroepiandrosterona (SDHEA), a androstenediona, a testosterona total e a testosterona livre. Foi utilizado como critério diagnóstico um nivel basal de $17 \mathrm{OHP}$ superior a $5 \mathrm{ng} / \mathrm{mL}$ e/ou valor de pico pós-ACTH superior a $10 \mathrm{ng} / \mathrm{ml}^{2}$. Os valores dos andrógenos considerados adequados para a faixa etária dos pacientes são os citados por Rosenfield's.

O eletrencefalograma (EEG) foi realizado da maneira tradicional em 1 paciente com idade inferior a 3 anos e pelo método quantitativo com mapeamento cerebral, utilizando o software Meditron, nos demais. A técnica utilizada e a interpretação de cada EEG seguiu uma rotina anteriormente descrita ${ }^{13}$. As frequências analisadas foram: delta $=1-3,5 \mathrm{~Hz}$; teta $=4-7,5 \mathrm{~Hz}$; alfa $=8-12,5 \mathrm{~Hz}$; beta $=13-32 \mathrm{~Hz}$. Resumidamente, foram considerados como representativos de anormalidade a presença de assimetrias ou de atividade paroxistica epileptogênica, alteraçðes da frequência, quer em relação à forma dominante ou à sua topografia, e a redução na porcentagem do ritmo alfa na regiăo occipital direita. A presença de ritmo alfa occipital foi considerada essencial nas crianças com 4 anos ou mais ${ }^{6}$. O predomínio de atividades lentas delta ou teta na áreas posteriores e a redução de $33 \%$ ou mais na porcentagem do ritmo alfa occipital à direita, caracterizaram os EEGs como "lentos".

\section{RESULTADOS}

Os dados clínicos e de maturação óssea dos pacientes estão discriminados na Tabela 1. Os 4 primeiros casos, com a forma clássica da doença, apresentaram genitália ambigua ao nascimento. Entre os pacientes com a forma não-clássica, 2 meninas tiveram como primeira manifestação clínica a pubarca, num dos casos aos 5 anos e, no outro, aos 6 anos e 2 meses, e a restante (Caso 7), hirsutismo aos 10 anos. Os 3 meninos se apresentaram com pubarca e macrogenitossomia, observados entre os 9 meses de idade e os 5 anos.

O mais baixo nivel de 170HP basal observado (Tabela 1) na ausência de tratamento foi de 7,4 $\mathrm{ng} / \mathrm{mL}$, enquanto o valor mais baixo de pico pós-ACTH foi de $15,1 \mathrm{ng} / \mathrm{mL}$. Na dosagem dos andrógenos, foi constatado valor aumentado de SDHEA em todos os pacientes, com exceção do Caso 7, de androstenediona em 3 (Casos 1,7 e 8), e de testosterona total em 4 (Casos 1,6,7 e 9). A testosterona livre foi dosada em 5 casos, estando elevada em 3 (Casos 1,7 e 8).

O exame neurológico tradicional não foi realizado em um dos pacientes (Caso 9), por falta de sua colaboração, e nas Pacientes 3 e 4, irmãs, por terem recusado acompanhamento neurológico. Nas outras crianças esse exame foi inalterado. Na história gestacional foram detectados: uso materno de carbamazepina, fenotiazínico e anticoncepcional oral (Caso 6), ameaças de aborto e tabagismo materno (Caso 9) e uso materno de álcool e outras drogas de abuso (Caso 10). Em 6 pacientes foram 
Tabela I-Dados clinicos, maturação óssea e niveis de 17-hidroxiprogestorena na apresentação de pacientes com hiperplasia supra-renal congènita.

\begin{tabular}{|c|c|c|c|c|c|c|c|c|}
\hline \multirow[t]{2}{*}{ Caso } & \multirow[t]{2}{*}{ HSRC } & \multirow[t]{2}{*}{ Sexo } & \multirow{2}{*}{$\begin{array}{c}\text { Idade } \\
\text { (anos,meses) }\end{array}$} & \multirow{2}{*}{$\begin{array}{c}\text { Altura } \\
\left({ }^{\circ} \mathrm{P}\right)\end{array}$} & \multirow{2}{*}{$\begin{array}{c}\text { Pelos } \\
\text { Pubianos** }\end{array}$} & \multirow[t]{2}{*}{$10^{* * *}$} & \multicolumn{2}{|c|}{$170 \mathrm{HP}(\mathrm{ng} / \mathrm{mL})$} \\
\hline & & & & & & & Basal & Pico Pós-ACTH \\
\hline 1. TSB & C-PS* & $\mathbf{F}$ & $4 a 6 m$ & $>97$ & III & A & $>25,0$ & - \\
\hline 2. ERS & C-PS* & $\mathbf{F}$ & $8 \mathrm{a} 3 \mathrm{~m}$ & $<3$ & V & C & 20,0 & - \\
\hline 3. AKCC & $C^{*}$ & $\mathbf{F}$ & $6 a$ & $3-10$ & II & $\mathrm{C}$ & 0,1 & - \\
\hline 4. $\mathrm{ACC}$ & $C^{*}$ & F & $14 a$ & $3-10$ & V & - & 4,2 & $\cdot$ \\
\hline 5. VNM & $\mathrm{NC}$ & F & $6 \mathrm{a} 8 \mathrm{~m}$ & $50-75$ & II & C & 12,9 & 41,5 \\
\hline 6. VFM & $\mathrm{NC}$ & $\mathbf{F}$ & $7 \mathrm{allm}$ & 75 & II & $\mathrm{C}$ & 18,8 & $>20,0$ \\
\hline 7. SMS & NC & $F$ & $12 \mathrm{a} 4 \mathrm{~m}$ & 90 & II-III & A & 13,0 & 70,0 \\
\hline 8. WRS & NC & $\mathbf{M}$ & lallm & $>97$ & III & A & 13,1 & 15,1 \\
\hline 9. $\mathrm{AJ}$ & $\mathrm{NC}$ & $\mathbf{M}$ & $6 \mathrm{a} 8 \mathrm{~m}$ & $>97$ & III & A & $>20,0$ & - \\
\hline 10. WB & $\mathrm{NC}$ & $\mathbf{M}$ & $7 \mathrm{a} 8 \mathrm{~m}$ & $25-50$ & II & A & 7,4 & 16,5 \\
\hline
\end{tabular}

HSRC, hiperplasia supra-renal congénita; C-PS, Clássica, perdedora de sal; C, clássica; NC, não-clássica.

* Avaliaça sob tratamento. $O$ Caso 1 utilizava apenas mineralocorticóide.

** Segundo Tanner ${ }^{3}$

*** Idade óssea: A, avançada; C, compativel. (de acordo com Pacientes e Métodos.)

obtidos dados referentes ao parto, sendo todos a termo, 2 cesáreas e ausência de história de baixo peso ao nascer ou outro acontecimento adverso perinatal. $O$ exame neurológico evolutivo detectou déficit na área de persistência motora que, associado a dados de história, foi compativel com o diagnóstico de hiperatividade e déficit de atenção em 2 pacientes dos 7 avaliados $(28,5 \%$; Casos $6 \mathrm{e}$ 10). No paciente masculino que não realizou exame neurológico, informaçðes sobre os primeiros anos de vida foram compatíveis com retardo de desenvolvimento neuropsicomotor.

Por ocasião do EEG, 6 pacientes estavam na vigência de tratamento para HSRC (Tabela 2), em 3 deles iniciado no período neonatal. Na época do exame, os valores basais da 170HP (dados ñ̃o apresentados) estavam excessivamente suprimidos - abaixo de $5 \mathrm{ng} / \mathrm{mL}^{12}$, em 2 pacientes (Casos 3 e 10), com supressão insatisfatória - acima de $10 \mathrm{ng} / \mathrm{mL}^{12}$, em 2 (Casos 2 e 8) e adequado nos restantes.

$O$ resultado final dos EEGs encontra-se na Tabela 2. Em 8 pacientes o resultado foi interpretado como anormal para a idade. No Caso 2 , o ritmo de base estava pouco organizado, com ritmo alfa predominando no limite inferior, $8,5 \mathrm{~Hz}$, ocupando pequena incidência (menos de $40 \%$ ), sendo pobremente modulado; também foi observado aumento moderado de frequências lentas teta nas áreas posteriores. No Caso 3, o EEG se mostrou lento, com predomínio das frequências teta $5 \mathrm{~Hz}$ nas áreas frontocentrais; nas regiठes occipitais o ritmo alfa ocupava apenas $20 \%$, com predomínio de frequências teta e delta. No Caso 4, o EEG foi discretamente lento, ritmo alfa com $40 \%$ nas áreas occipitais, e frequências teta um pouco aumentadas nas áreas centrais e posteriores. No Caso 5 , foi detectada atividade de base lenta, existindo nas áreas occipitais um aumento das frequências teta e redução do ritmo alfa. No Caso 6 , foi observada redução do ritmo alfa occipital e aumento moderado das frequências lentas teta, com aparente aumento difuso da atividade delta. No Caso 7, o traçado apresentou atividade de base desorganizada, com trechos de ritmo alfa mal modulados, embora na faixa superior desta banda de frequência (11 a $12 \mathrm{~Hz}$ ); dados quantitativos mostraram pequena incidência de ritmo alfa nas áreas occipitais e aumento das frequências lentas teta. Foram também registrados sinais irritativos com descarga de ponta-onda nas áreas anteriores, iniciando à direita, na 
Tabela 2 - Achados Eletrencefalográficos em crianças com hiperplasia supra-renal congênita por deficiência da 21-Hidroxilase.

\begin{tabular}{lccc}
\hline Caso & Idade na época do EEG & Tempo de uso de glicocorticóide & Diagnóstico eletrencefalográfico \\
\hline 1. TSB & $4 \mathrm{a} 11 \mathrm{~m}$ & 5 meses & Normal \\
2. ERS & $15 \mathrm{a} 5 \mathrm{~m}$ & semrpe & lento \\
3. AKCC & $12 \mathrm{a} 5 \mathrm{~m}$ & sempre* & lento \\
4. ACC & $18 \mathrm{a} 5 \mathrm{~m}$ & sempre & lento \\
5. VFM & $8 \mathrm{a} 5 \mathrm{~m}$ & zero & lento \\
6. VNM & $7 \mathrm{a} 2 \mathrm{~m}$ & zero & lento \\
7. SMS & $12 \mathrm{a} 5 \mathrm{~m}$ & zero & lento e paroxistico \\
8. WRS & $2 \mathrm{a} 4 \mathrm{~m}$ & 5 meses & normal** \\
9. AJ & $6 \mathrm{a} 8 \mathrm{~m}$ & zero & lento e assimétrico \\
10. WB & $10 \mathrm{a} 11 \mathrm{~m}$ & 2 anos & paroxístico \\
\hline
\end{tabular}

*Desde o diagnóstico, no periodo neonatal.

** Realizado durante sono induzido.

região frontal, e aparecendo a seguir na região frontal esquerda. No Caso 9 , foi observada redução do ritmo alfa na região occipital esquerda em porcentagem e amplitude, e aumento da frequência teta na região occipital esquerda. No Caso 10, a atividade de base foi normal para a idade, com predomínio de ritmo occipital e sem assimetrias, mas com sinais irritativos na região temporal direita.

\section{DISCUSSÃO}

A pubarca, nos casos desta série, é uma apresentação clássica da forma tardia da HSRC por deficiência da 21 -hidroxilase. Os níveis de $17 \mathrm{OHP}$, basais e pós-estímulo, são similares aos observados por outros autores ${ }^{11,14}$.

Chama a atenção em nossos resultados a presença de concentrações aumentadas de SDHEA na quase totalidade dos pacientes, enquanto que a androstenediona mostrou-se elevada em apenas 3. O SDHEA não tem sido recomendado como marcador diagnóstico da HSRC ${ }^{\prime}$. Por sua vez, o aumento da androstenediona foi constante nos 11 pacientes com a forma não-clássica da deficiência da 21 hidroxilase, 3 dos quais com pubarca precoce, no relato de Elias e Moreira ${ }^{3}$.

Em relação ao tratamento da HSRC, os controles obtidos em nossos pacientes na época em que realizaram o EEG demonstram as dificuldades no manuseio terapêutico. $O$ glicocorticóide aqui utilizado foi a prednisona. Esta opçâo se baseia na dificil aderência ao tratamento comumente associada ao uso de hidrocortisona via oral, corticóide esse que exige um fracionamento diário em 3 doses, em função da sua meia-vida curta. A dose preconizada na literatura e aqui empregada foi de aproximadamente $6 \mathrm{mg} / \mathrm{m}^{2} / 24 \mathrm{~h}^{10}$. As duas pacientes desta série com a forma clássica não perdedora de sal não utilizavam mineralocorticóide, o qual poderia ser indicado em função da atividade antagonista aos mineralocorticóides e consequente aumento da atividade da renina plasmática e do ACTH, apresentadas pela $170 \mathrm{HP}$ e pela progesterona. Uma dessas pacientes apresentava controle adequado da doença.

Os resultados da avaliação eletrencefalográfica, alterados em $80 \%$ da amostra, com EEGs caracterizados como lentos em 7 pacientes, chama a atenção. Esse padrão é sugestivo da presença atual ou passada de patologia neurológica ${ }^{6}$. Lesões neurológicas associadas a trauma de parto não 
parecem ser a etiologia das alterações aqui detectadas, visto os dados da avaliação neurológica evolutiva. Outra possibilidade é a de que esses achados sejam secundários aos níveis excessivos de andrógenos e/ou à precocidade com que ocorreu o aumento desses níveis. Explicação similar, isto é, elevação prematura da secreção de andrógenos, seria operante nos casos de pubarca, nos quais as mesmas anormalidades eletrencefalográficas são encontradas.

Em animais de experimentação, a testosterona causa efeito direto e específico no sistema nervoso central, interferindo com o periodo refratário neuronal, por alteraçóes na membrana celular?. A progesterona também interage com receptores de membrana, alterando a liberação de substâncias neuroativas e o fluxo de cloro?. Além das ações de membrana, os esteróides adrenais afetam a sobrevivência neuronal e a estrutura dendrítica no hipocampo, regulando vários processos neuroquímicos, efeitos esses que resultam da ação sobre a expressão de gens ${ }^{9}$.

Em humanos há evidências de que os andrógenos possuam, ao nível do sistema nervoso central, ações similares às das drogas psicotrópicas ${ }^{5}$.

Independentemente do mecanismo fisiopatológico envolvido nas alteraçōes observadas neste estudo os autores concluem sobre a importância da avaliação e acompanhamento neurológico em crianças com HSRC.

\section{REFERÊNCIAS}

1. Azziz R, Dewailly D, Owerbach D. Nonclassic adrenal hyperplasia: current concepts. J Clin Endocrinol Metab 1994;78:810-815.

2. Dewailly D, Vantyghem-Haudiquet MC, Sainsard C, Buvat J, Cappoen JP, Ardaens $K$ et al. Clinical and biological phenotypes in late-onset 21-hydroxylase deficiency. J Clin Endocrinol Metab 1986;63:418-423.

3. Elias LLK, Moreira AC. Aspectos clínicos e laboratoriais da forma não-clássica da hiperplasia adrenal congênita por deficiência da 21 -hidroxilase. Arq Bras Endocrinol Metab 1992;36:72-75.

4. Greulich WW, Pyle SI. Radiographic atlas of skeletal development of the hand and wrist. Ed 2. California: Stanford Univ Press, 1974.

5. Hermann WM, Beach RC. Psychotropic effects of androgens: a review of clinical observations and new human experimental findings. Pharmakopsych 1976;9:205-219.

6. Kellaway P. An orderly approach to visual analysis: characteristics of the normal EEG of adults and children. In Daly D, Pedley T. (eds). Current practice of clinical electroencephalography. New York: Raven Press, 1990:139-199.

7. Kendrick KM. Electrophysiological effects of testosterone on the medial preoptic-anterior hypothalamus of the rat. J Endocr 1983;96:35-42.

8. Liu N, Grumbach MM, De Napoli RA, Morishima A. Prevalence of electroencephalographic abnormalities in idiopathic precocious puberty and premature pubarche: bearing on pathogenesis and neuroendocrine regulation of puberty. J Clin Endocr 1965;25:1296-1308.

9. McEwen BS. Our changing ideas about steroid effects on an ever-changing brain. Neurosciences 1991;3:497-507.

10. Migeon CJ, Donohoue PA. Adrenal disorders. In Kappy MS, Blizzard RM, Migeon CJ, (eds). The diagnosis and treatment of endocrine disorders in childhood and adolescence. Springfield: Charles $C$ Thomas, 1994:717-856.

11. Moreira AC, Elias LLK. Pituitary-adrenal responses to corticotropin-releasing hormone in different degrees of adrenal 21-hydroxylase deficiency. J Clin Endocrinol Metab 1992;74:198-203.

12. New MI. Steroid 21-hydroxylase deficiency (congenital adrenal hyperplasia). Am J Med 1995; 98 (Suppl 1A): $1 \mathrm{~A}-2 \mathrm{~S}$

13. Oliveira MC, Fernandes MH, Barea LM, Pereira-Lima J, Hoffmann K, Puiatti D et al. Achados neurológicos em crianças com várias apresentaçðes de precocidade sexual. Arq Bras Endocrinol Metab 1994;38:146-154.

14. Pang S, Clark A. Congenital adrenal hyperplasia due to 21-hydroxylase deficiency: newborn screening and its relationship to the diagnosis and treatment of the disorder. Screening 1993;2:105-139.

15. Rosenfield RL. The ovary and female sexual maturation. In Kaplan SA (ed). Clinical pediatric endocrinology. Philadelphia: WB Saunders, 1990:259-323.

16. Tanner JM, Whitehouse RH. Clinical longitudinal standards for height, weight, height velocity, weight velocity, and the stages of puberty. Arch Dis Child 1976;51:170-179. 